\title{
Insights into shell deposition in the Antarctic bivalve Laternula elliptica: gene discovery in the mantle transcriptome using 454 pyrosequencing
}

\author{
Melody S Clark*1, Michael AS Thorne1, Florbela A Vieira², João CR Cardoso², Deborah M Power² and Lloyd S Peck'
}

\begin{abstract}
Background: The Antarctic clam, Laternula elliptica, is an infaunal stenothermal bivalve mollusc with a circumpolar distribution. It plays a significant role in bentho-pelagic coupling and hence has been proposed as a sentinel species for climate change monitoring. Previous studies have shown that this mollusc displays a high level of plasticity with regard to shell deposition and damage repair against a background of genetic homogeneity. The Southern Ocean has amongst the lowest present-day $\mathrm{CaCO}_{3}$ saturation rate of any ocean region, and is predicted to be among the first to become undersaturated under current ocean acidification scenarios. Hence, this species presents as an ideal candidate for studies into the processes of calcium regulation and shell deposition in our changing ocean environments.
\end{abstract}

Results: 454 sequencing of L. elliptica mantle tissue generated 18,290 contigs with an average size of 535 bp (ranging between $142 \mathrm{bp}-5.591 \mathrm{~kb}$ ). BLAST sequence similarity searching assigned putative function to 17\% of the data set, with a significant proportion of these transcripts being involved in binding and potentially of a secretory nature, as defined by GO molecular function and biological process classifications. These results indicated that the mantle is a transcriptionally active tissue which is actively proliferating. All transcripts were screened against an in-house database of genes shown to be involved in extracellular matrix formation and calcium homeostasis in metazoans. Putative identifications were made for a number of classical shell deposition genes, such as tyrosinase, carbonic anhydrase and metalloprotease 1, along with novel members of the family 2 G-Protein Coupled Receptors (GPCRs). A membrane transport protein (SEC61) was also characterised and this demonstrated the utility of the clam sequence data as a resource for examining cold adapted amino acid substitutions. The sequence data contained 46,235 microsatellites and 13,084 Single Nucleotide Polymorphisms(SNPs/INDELS), providing a resource for population and also gene function studies.

Conclusions: This is the first 454 data from an Antarctic marine invertebrate. Sequencing of mantle tissue from this non-model species has considerably increased resources for the investigation of the processes of shell deposition and repair in molluscs in a changing environment. A number of promising candidate genes were identified for functional analyses, which will be the subject of further investigation in this species and also used in model-hopping experiments in more tractable and economically important model aquaculture species, such as Crassostrea gigas and Mytilus edulis.

\section{Background}

Laternulids are infaunal bivalve molluscs, which morphologically resemble the soft-shelled clam Mya arenaria, the major ingredient of clam chowder. In spite of a widespread latitudinal distribution ranging from the tropics, through temperate Australasia to Antarctica [1], research

* Correspondence: mscl@bas.ac.uk

${ }^{1}$ British Antarctic Survey, Natural Environment Research Council, High Cross, Madingley Road, Cambridge, CB3 OET, UK

Full list of author information is available at the end of the article on this genus is dominated by work on the Antarctic species (Laternula elliptica). This clam has been studied for a number of years and is one of the best characterised Antarctic marine invertebrates. Studies initially focused on its ecology [2], and general physiology: reproduction $[3,4]$, development $[5,6]$, growth $[7,8]$ and seasonal energetics $[9,10]$. However more recent research has focused on the longevity of this species in relation to reactive oxygen species production, antioxidant defences and cellular ageing [11], as this species often lives 25 years or more 
[12]. It has also been the subject of significant investigation of its thermal tolerance and the expected impact of climate change [13-15].

Antarctic marine invertebrates are stenothermal [14] and L. elliptica is one of the more sensitive species [1316]. These animals suffer significant mortalities at $4-5^{\circ} \mathrm{C}$, but lose essential biological functions, such as the ability to bury in sediment, much earlier, at only $1-2^{\circ} \mathrm{C}$ over current summer maximum sea water temperatures [13-15]. This thermal response is viewed against predictions that globally oceanic sea surface temperatures are predicted to rise on average by $2^{\circ} \mathrm{C}$ over the next 100 years $[17,18]$. However, regional differences are apparent and climate change along the Antarctic Peninsula has been particularly rapid with a temperature increase in the surface layers of the Bellingshausen Sea of $1^{\circ} \mathrm{C}$ in 50 years [19]. The predictions of the effect of these thermal changes on Antarctic marine biodiversity are complex [20] and further complicated by reductions in ocean $\mathrm{pH}$.

Antarctic species, in general, have been proposed as excellent candidates for the development of climate change molecular biomarkers [21], whilst L. elliptica in particular has strong support as a sentinel species [22]. It has a circumpolar distribution and is highly abundant [23]. It is the largest individual mollusc in terms of live weight [7] and one of the highest in terms of total ecological biomass [24]. Being an infaunal filter-feeder, it also plays a significant role in benthopelagic coupling $[25,26]$ and therefore is a keystone species of the Antarctic marine ecosystem.

In the 250 years since the onset of the industrial revolution, ocean $\mathrm{pH}$ has fallen from an average of 8.16 to 8.05 and is predicted to decrease by a further $0.3-0.4 \mathrm{pH}$ units by the end of this century. These predicted changes in ocean $\mathrm{pH}$ are greater, and far more rapid, than any experienced in the past 300 million years [27-30]. The Southern Ocean will be particularly affected as it has amongst the lowest present-day $\mathrm{CaCO}_{3}$ saturation state of any ocean region, and will therefore be among the first to become undersaturated [31]. The ability of marine organisms to adapt to this unprecedented environmental modification of increased temperature and reduced $\mathrm{pH}$ is largely unknown, with particular concern expressed over calcifying animals such as echinoderms and molluscs [27].

Laternula species are conservative in shell form and habitat suggesting they share morphological constraints at different latitudes [32]. Recent data suggests however, that at least in the Southern Ocean, L. elliptica exhibits a high degree of plasticity in the thickness of shell deposition. This physical characteristic varies markedly between locations along the Antarctic Peninsula. Speci-

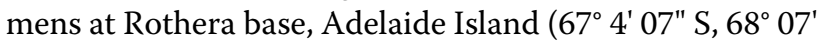
$30 \mathrm{~W}$ W) have shells at least 2-3× thicker than those at Jubany base King George Island $\left(62.23^{\circ} \mathrm{S}, 58.67^{\circ} \mathrm{W}\right)$
(Harper, pers comm). The Rothera animals also have damage repair rates 5-12× higher than Jubany animals, possibly due to increased frequency of iceberg impact (Harper, pers comm). This plasticity in shell thickness is superimposed against a homogeneous population structure across all sites (Hoffman, pers comm). Such plasticity in shell deposition has also been observed in the Antarctic limpet, Nacella concinna [33].

Shells of $L$. elliptica were recently subjected to an end of century scenario $\mathrm{pH} 7.4$ trial and showed extensive damage over 56 days [34]. However, these were isolated shells and such studies do not take into account the ability of the animal to ameliorate shell loss via increased deposition or the effects of having an infaunal life habit. To understand the dynamics of shell turnover and the response of this process to both biotic and abiotic factors a better understanding of the molecular basis and regulation of shell formation is required. The studies which do exist have revealed that the molluscan shell is composed largely of calcium carbonate and organic macromolecules which are secreted by the mantle [35-40]. Hence, whole animal studies are essential not only to understand the shell deposition process in relation to altered temperature and $\mathrm{pH}$, but also the more subtle effects of altered environmental conditions on calcium regulation in cellular processes and the energetic trade-offs of responses to climate change.

The aragonitic shell of L.elliptica comprises two layers: a very thin prismatic layer on the outside covering layers of sheet nacre on the inner side (with sheet nacre on both sides of the pallial myostracum); and a periostracum of around $10 \mu \mathrm{m}$ surrounds the outer shell where it has not been removed by abrasion [[41,42]; Harper, pers comm.]. The mantle secretes the shell and forms a large thin sheet of tissue between the shell and the internal organs and extends beyond the rest of the body, so that part of the edge is exposed to external conditions [42]. It thus serves two purposes, secretion of the shell matrix and also protection from the external environment, either via sealing the edge of the shell or from damage to the shell itself (for example, after being impacted by an iceberg (Peck, pers comm)). The edge of the mantle comprises three folds, of which, only the outer fold is involved in laying down the actual shell material. The periostracum emerges from the groove between the middle and outer folds and bends back to cover the shell. The primary role of the perisotracum is believed to be shell secretion and maintenance, but there are other roles such as protection from the external environment, infestation and predatory borers [42]. The mantle tissue for this analysis was taken as a cross section of all mantle folds and included periostracum material.

So far molecular work in L. elliptica has been limited to candidate genes concerned with understanding thermal 
tolerance via antioxidants and heat shock proteins [[4345], Truebano et al, submitted]. The aim of this study is to develop molecular resources for this species. We focus on the mantle, as it is the main shell secreting organ (Figure 1 ) and we are particularly interested in the processes of calcification and comparative ossification [46-48]. 454 pyrosequencing technologies enable the rapid generation of transcriptomes for non-model species [49]. This approach is exploited in the present study, the results of which will be the basis of future studies of calcium regulation in L. elliptica in relation to environmental change. Here we describe the transcriptome of the mantle tissue of L. elliptica, focussing on the datamining of genes involved in calcium regulation and shell deposition. This represents the first publicly available 454 data for an Antarctic marine invertebrate and provides an important comparative resource for such studies in more eurythermal temperate mollusc species.

\section{Results and Discussion}

The non-normalised laternula libraries were subjected to a full 454 run that yielded 1,034,155 reads totalling $381,838,384$ bases with an average read length per trancript of 369 bases. After cleaning the data and removing small reads, 778,629 reads with an average size of $304 \mathrm{bp}$ were entered into Newbler for assembly. These assembled into the 18,290 contigs (264,289 reads) which were used for further analysis. Because the aim of this project was to identify and characterise specific genes for future analyses, in particular the GPCRs and several gene families, such as collagen and bone morphogenic proteins (Additional files: Tables S1 and S2) there was a requirement for longer sequences of good quality which would enable us to distinguish between gene family members. Hence the descriptive analysis presented here utilised only the contigs produced by the assembly. Whilst the singletons potentially contain useful lowly expressed sequences, they also contain a substantial proportion of artefacts derived from cDNA synthesis, sequencing and contamination [50]. PCR and re-sequencing of singletons

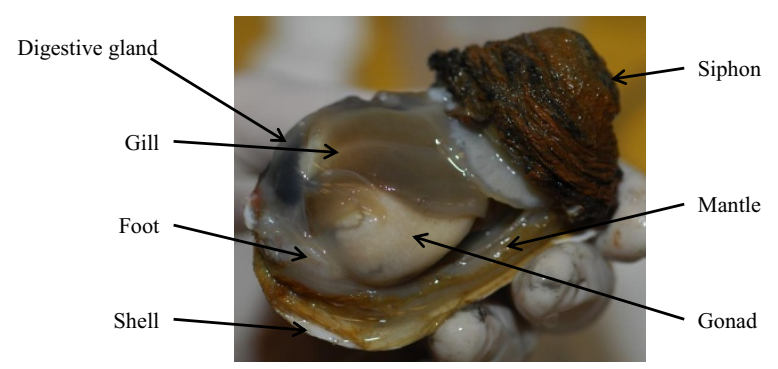

Figure 1 Annotated longitudinal dissection of L. elliptica with one of the shells removed. Photograph copyright permission obtained from Erwan Amice. is essential in order to verify the gene products [50]. The contigs ranged in size from $142 \mathrm{bp}$ to $5591 \mathrm{bp}$, with an average size of $535 \mathrm{bp} .42$ contigs were greater than $3 \mathrm{~kb}$ and 69 contigs comprised more than 300 reads, with the largest contig of $5591 \mathrm{bp}$ comprising the most reads with 1000 sequences (Table 1). Self BLAST of this dataset produced only 281 matches with a value of $\mathrm{e}^{-100}$, indicating a low level $(<1.5 \%)$ of redundancy in the assembly of the reads. The contigs contained 46,235 microsatellites, of which 1,608 comprised over 7 repeat units (Additional File Table S3). There were 13,084 SNPs/INDELS present in 2,475 contigs designated as high confidence by the Newbler program (Additional file Table S4), although a further circa 25,000 SNPs were identified at lower confidence level as defined by Newbler [51]. These figures for microsatellite and SNP/INDEL detection are at a similar relative level to those identified in the transcriptome of another non-model species, the flesh fly (Sarcophaga crassipalpis) [52]. Given that L. elliptica is a wild-caught species and the S. crassipalpis material came from a long standing inbred laboratory stock, higher levels of these genetic variants would be expected in the clam. However, analysis in this species was restricted to contigs only, potentially reducing the dataset.

Sequence similarity searching of the GenBank nonredundant database with BLAST produced matches against only 3,098 of the contigs using a $<1 \mathrm{e}^{-10}$ cut off value. This poor level of sequence similarity matching $(17 \%)$ has also been noted in previous work on this species (Truebano et al, submitted) and is due to a lack of sequences from a closely related mollusc species in the databases. This is reflected in the number of different species that show sequence matches against our data; Table 1 comprises 39 BLAST sequence similarity results with the best matches originating from 33 species ranging from hydrozoans and arthropods through to vertebrates. To date $(25 / 01 / 10)$ there are only 25,032 nucleotide sequences, 195,275 ESTs, 14,507 proteins and 356 genes from the class Bivalvia in the public databases http://www.ncbi.nlm.nih.gov and these are dominated by entries from Mytilus and Crassostrea species. At the subclass level, the number of nucleotide and protein entries are 86 and 19 respectively, which is further reduced to 24 and 16 at the family level. The genbank non-redundant database [53] is one of the best annotated sources for comparative in silico gene analyses. However, of potential use, in terms of EST verification and gene mining are other less well annotated sources of molluscan sequence data, such as the sequenced genome of the gastropod snail (Lottia gigantea) and 454 data from Mytilus species [54]. These comprise larger molluscan datasets than found in genbank, but BLAST sequence similarity searches using a $<1 \mathrm{e}-10$ cut off value merely emphasized the evolutionary distance between the molluscs studied. 
Table 1: Most commonly expressed sequences with associated BLAST matches.

\begin{tabular}{|c|c|c|c|c|c|c|}
\hline Contig ID & Length (bp) & No of reads & Description & Species & Common name & E-value \\
\hline 00447 & 5591 & 1000 & $\begin{array}{l}\text { Map kinase interacting serine } \\
\text { threonine protein kinase. }\end{array}$ & Aplysia californica & California sea hare & $1.0 \mathrm{e}-148$ \\
\hline 00731 & 2559 & 697 & Collagen pro-a chain & Haliotis discus & Pacific abalone & 9.5 e-31 \\
\hline 00765 & 1668 & 647 & Enolase & Loligo pealei & Long-finned squid & $1.5 \mathrm{e}-184$ \\
\hline 17466 & 2025 & 547 & ATP synthase sub-unit a & Pinctada fucata & Pearl oyster & 1.9 e-243 \\
\hline 02034 & 1166 & 544 & Collagen type IV a6 & Ciona intestinalis & Sea squirt & $2.0 \mathrm{e}-13$ \\
\hline 17241 & 1652 & 544 & Troponin T & Patinopecten yessoensis & Yesso scallop & 1.9 e-32 \\
\hline 16715 & 1674 & 505 & B cell translocation gene & Crassostrea gigas & Pacific oyster & 2.1 e-36 \\
\hline 17817 & 3029 & 477 & Poly adenylate binding protein & Bos taurus & Cow & 7.6 e-196 \\
\hline 17035 & 3832 & 466 & Phosphoenolpyruvate carboxylase & Crassostrea gigas & Pacific oyster & $1.1 \mathrm{e}-270$ \\
\hline 00554 & 1333 & 455 & Ornithine decarboxylase & Haliotis diversicolor & Abalone & 6.9 e-63 \\
\hline 01359 & 2332 & 449 & Tyrosinase & Sepia officinalis & Cuttlefish & $9.3 \mathrm{e}-47$ \\
\hline 01057 & 2938 & 438 & Arginine kinase & Carbicula japonica & Shijimi clam & 5.1 e- 267 \\
\hline 00449 & 1698 & 428 & $\begin{array}{l}\text { Voltage gated potassium channel } \\
\text { complex }\end{array}$ & Mus musculus & Mouse & $7.2 \mathrm{e}-11$ \\
\hline 17467 & 535 & 421 & $\begin{array}{l}\text { Stress associated endoplasmic } \\
\text { reticulum protein (SERP2) }\end{array}$ & Bos taurus & Cow & 3.0 e-21 \\
\hline 00029 & 2433 & 411 & Transport protein SEC1 subunit a & Culex quinquefasciatus & Mosquito & 4.4 e-235 \\
\hline 01548 & 1543 & 405 & Calponin/transgelin & Haliotis discus & Pacific abalone & 1.7 e-34 \\
\hline 00054 & 1486 & 399 & $\begin{array}{l}\text { Mitochondrial carrier protein, } \\
\text { putative ADP/ATP translocase }\end{array}$ & Lepeophtheirus salmonis & Copepod sea louse & 7.3 e-112 \\
\hline 00562 & 3798 & 396 & Thymosin $\beta$ & Triatoma infestans & $\begin{array}{l}\text { Chagas insect } \\
\text { disease vector }\end{array}$ & 4.0 e-19 \\
\hline 00500 & 1985 & 395 & $\begin{array}{l}\text { Adipose differentiation-related } \\
\text { protein }\end{array}$ & Anas platyrhynchos & Mallard duck & 2.5 e-42 \\
\hline 00119 & 966 & 388 & 40 s ribosomal protein $\mathrm{S} 2$ & Urechis caupo & Echiuran worm & $1.2 \mathrm{e}-109$ \\
\hline 02431 & 804 & 382 & $60 \mathrm{~s}$ ribosomal protein L15 & $\begin{array}{l}\text { Ctenopharyngodon } \\
\text { idella }\end{array}$ & Grass carp & 7.8 e-76 \\
\hline
\end{tabular}


Table 1: Most commonly expressed sequences with associated BLAST matches. (Continued)

\begin{tabular}{|c|c|c|c|c|c|c|}
\hline 00103 & 2028 & 374 & NADH-ubiquinone oxidase & $\begin{array}{l}\text { Lophiotoma } \\
\text { cerithiformis }\end{array}$ & $\begin{array}{l}\text { Conoidean } \\
\text { gastropod }\end{array}$ & 6.6 e-65 \\
\hline 01042 & 1310 & 359 & Y-box factor homolog & Aplysia californica & California sea hare & $6.1 \mathrm{e}-43$ \\
\hline 00753 & 3170 & 357 & Vacuolar ATP synthase & Salmo salar & Atlantic salmon & $2.2 \mathrm{e}-49$ \\
\hline 00168 & 1820 & 356 & Myosin heavy chain & Mytilus galloprovincialis & $\begin{array}{l}\text { Mediteranean } \\
\text { mussel }\end{array}$ & 1.6 e-191 \\
\hline 17000 & 912 & 354 & Ribosomal protein L3 & Spodoptera frugiperda & Fall armyworm & $3.9 e-113$ \\
\hline 00730 & 1839 & 351 & ATP synthase sub unit $\beta$ & Pinctada fucata & Pearl oyster & 1.5 e-111 \\
\hline 17045 & 513 & 341 & Ribosomal protein L28 & Sipunculus nudus & Marine worm & $2.2 \mathrm{e}-39$ \\
\hline 16762 & 1674 & 325 & Ubiquitin-conjugating enzyme & $\begin{array}{l}\text { Rhipicephalus } \\
\text { sanguineus }\end{array}$ & Brown dog tick & $2.4 \mathrm{e}-13$ \\
\hline 01081 & 1740 & 325 & Calponin & Mytilus galloprovincialis & $\begin{array}{l}\text { Mediteranean } \\
\text { mussel }\end{array}$ & 6.4 e-51 \\
\hline 01704 & 1585 & 324 & a macroglobulin & $\begin{array}{l}\text { Macrobrachium } \\
\text { rosenbergii }\end{array}$ & Giant river prawn & $1.6 \mathrm{e}-40$ \\
\hline 00954 & 1609 & 323 & Catalase & Chlamys farreri & Japanese scallop & 7.9 e-216 \\
\hline 01079 & 2064 & 318 & Troponin & Patinopecten yessoensis & Yesso scallop & 3.4 e-36 \\
\hline 01055 & 4390 & 317 & $\begin{array}{l}\text { Mannan-binding lectin-associated } \\
\text { serine protease }\end{array}$ & Cyprinus cario & Common carp & 4.4 e-32 \\
\hline 05926 & 594 & 313 & 40 s ribosomal protein $\mathrm{S} 11$ & Lineus viridis & Nemertean & 3.3 e-61 \\
\hline 00567 & 614 & 311 & $\begin{array}{l}\text { GABA }(A) \text { receptor associated } \\
\text { protein }\end{array}$ & Brachiostoma belcheri & Amphioxus & 7.7 e-57 \\
\hline 17744 & 1394 & 309 & Prosaposin & Danio rerio & Zebrafish & $1.8 \mathrm{e}-10$ \\
\hline 17082 & 604 & 307 & Actin & Podocoryne carnea & Hydrozoan & $3.2 \mathrm{e}-42$ \\
\hline 00083 & 758 & 306 & Fructose-biphosphate aldolase & Haliotis discus & Pacific abalone & 8.0 e-89 \\
\hline
\end{tabular}

For example, just over $2 \%$ of the Laternula contigs matched the ESTs and EST clusters produced from Lottia, although this increased to $17.5 \%$ against the Lottia filtered gene set. Less than $1 \%$ of the Laternula contigs matched the Mytilus mantle-specific 454 libraries and the 42,364 ESTs from M. californianus in GenBank. Hence there are no species closely related to L. elliptica with large amounts of sequence data in the public domain and therefore our data significantly increases resources in this area and provides an important source of comparative data for other Molluscan species.

\section{Highly expressed sequences}

The most commonly expressed genes in the Laternula dataset comprise various functional classes, which is 
reflected in the overall GO classifications (Figure 2). As stated previously, the edge of the mantle comprises three folds and the periostracum with the tissue for this transcriptome analysis taken from a cross section across all layers. BLAST sequence similarity searches revealed a wide range of diverse functions among the most commonly expressed genes (contigs comprising over 300 individual sequences) (Table 1) reflecting the complex contractile and secretory nature of this organ.

The mantle, whilst not a muscle per se, is contractile and hence many of the highly expressed sequences consist of structural or muscle-related genes, such as actin, collagen, troponin, calponin, adipose differentiationrelated protein and myosin [55], although some e.g. collagen, may also be involved in shell synthesis [56]. Interestingly, the most commonly expressed sequence is that of a MAP kinase interacting serine threonine protein kinase (Mnk1). This gene is a transcriptional and translational regulator of mRNA, in particular acting via the phosphorylation of the elongation initiation factor (EIF4E), which is an important modulator of cell growth and proliferation [57]. Studies in Aplysia have shown Mnk1 to be a negative regulator of cap-dependant translation in neurons [58], whilst in other species it has also been shown to bind stress activated p38 and may play a role in response to environmental stress [59]. The role of this gene in cell

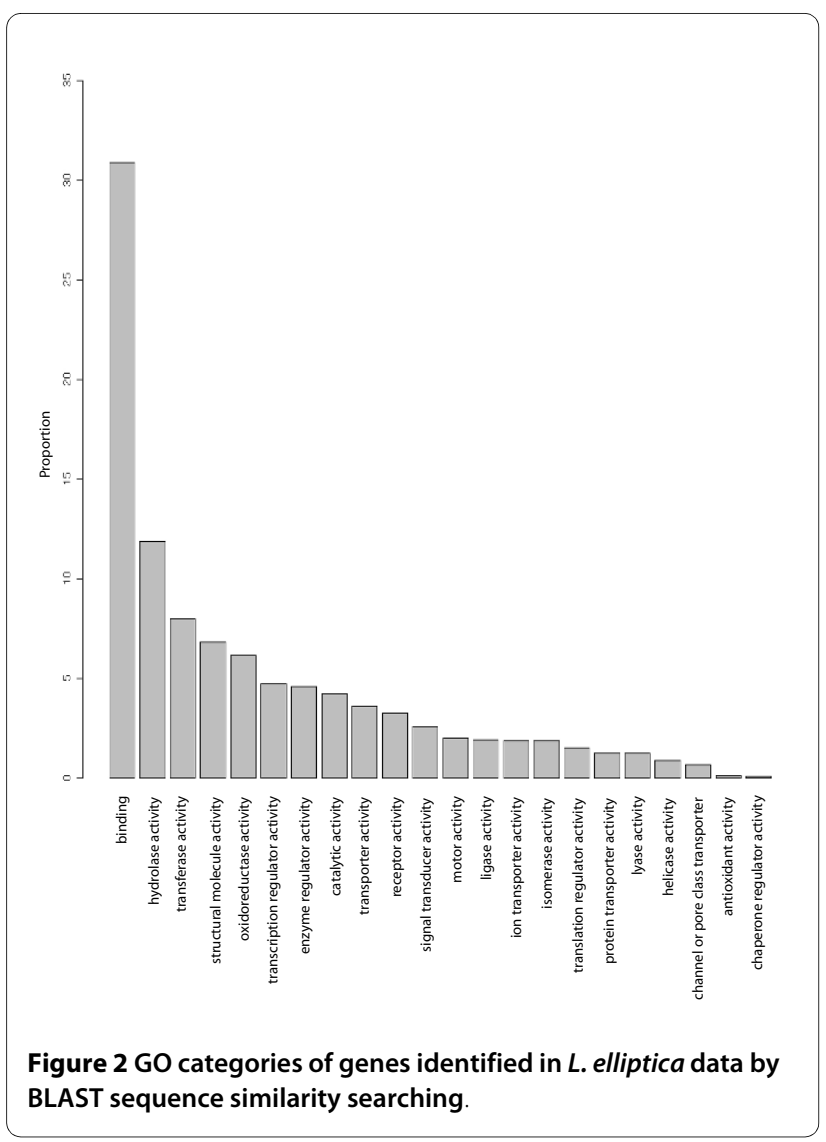

growth links with the identification of the B cell translocation gene (also involved in cell differentiation) and the Y-box factor homologue (a transcriptional and translational regulator of mRNA) [60], indicating that the mantle is an area of continual growth.

From the above, the mantle is clearly a metabolically and transcriptionally active tissue. This is further exemplified by the presence of ATP synthases, an ADP/ATP translocase, NADH-ubiquinone oxidase, genes from the glycolysis pathway, ribosomal RNAs and arginine kinase. The latter is a phosphagen kinase and these enzymes are prevalent in systems with fluctuating energy demands, acting as an energy buffering system [61] and also as an energy shuttle delivering ATP generated by mitochondria to high energy requiring processes, such as membrane turnover and potentially shell deposition [62]. Whilst the phosphagen kinases are a multigene family, arginine kinase is the only form of this gene in arthropods and molluscs [63]. It also has other functions such as buffering intracellular $\mathrm{pH}$ which would be important in the extrapallial space with the supersaturation of shell matrix components, including calcium ions.

The secretory nature of the mantle tissue requires a number of membrane transport proteins, represented in our limited identifications by a component of a voltage gated potassium channel complex, a V-type ATP synthase, which may transport solutes and lower $\mathrm{pH}$ in organelles, prosaposin and the endoplasmic reticulum transport protein SEC61 $\alpha$ sub-unit. The latter protein has been functionally studied in yeast and shown to play a crucial role in translocation of secretory polypeptides across the endoplasmic reticulum membrane [64]. Protein alignments of SEC61 $\alpha$ sub-unit from cold (Polar) and temperate fish species identified a number of putative cold adaptive amino acid substitutions [64]. The $L$. elliptica data contained the full length sequence of this gene and alignment with other species, specifically the fish forms [64] showed that L. elliptica does not have the proposed teleost cold water-specific amino acid modifications at positions 327, 328 and 339 in the loop between transmembrane regions 7 and 8 (Figure 3). One hypothesis, at the time, was that these changes were not adaptive, but inherited from a common fish ancestor and our data would appear to substantiate the latter hypothesis. Indeed over the stretch of 120 amino acids shown (Figure 3 ), the addition of $L$. ellipitica, the temperate bivalve $L$. gigantea and three insects to the fish alignment [64] indicates that within this stretch alone, there are 11 putative invertebrate-specific substitutions, 3 substitutions specific to the insects and one restricted to the 2 mollusc species. The implications of these changes cannot be quantified without functional studies, however, our Antarctic invertebrate dataset provides a significant resource 


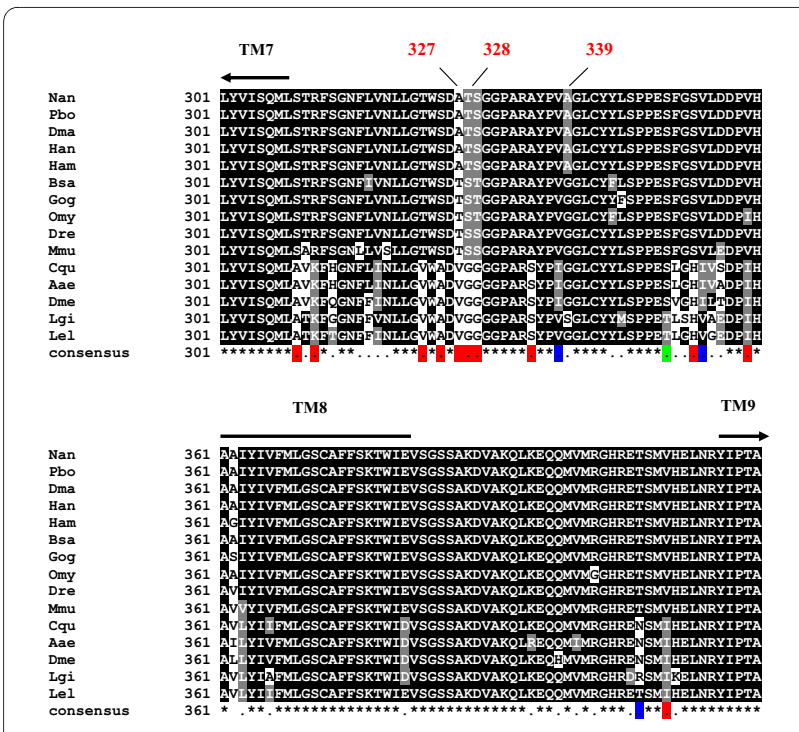

Figure 3 Amino acid alignment of the region between transmembrane (TM) regions 7 and 9 of the a sub-unit of SEC61. Putative cold-adapted amino acid substitutions are indicated at positions 327 , 328 and 339. Invertebrate-specific substitutions are labelled in red on the consensus line, insect-specific substitutions labelled in blue and the single potential bivalve-specific substitution labelled in green. Species abbreviations and accession numbers: Nan: Notothenia angustrata (Q8AY35); Pbo: Pagothenia borchgrevinki (Q8AY36); Dma: Dissostichus mawsoni (AY113841); Han: Harpagifer antarcticus (Q7T278); Ham: Hemitripterus americanus (Q8AY34); Bsa: Boreogadus saida (Q8AY33); Gog: Gadus ogac (Q8AY32) (all cold-adapted); Omy: Onchorhynchus mykiss (Q98SN9); Dre: Danio rerio (Q90ZM2); Mmu: Mus musculus (P61620); Cqu: Culex quinquefasciatus (BOWNA0); Aae: Aedes aegypti (Q17CM3); Dme: Drosophila melanogaster (Q8STG9). Lgi: Lottia gigantea (cluster: >jgi|Lotgi1|194715|estExt_Genewise1.C_sca_610223 from sequences: 4236761:1772, 4236761:2059 and 4236761:4476 extracted from http:// genome.jgi-psf.org/Lotgi1/Lotgi1.home.html; Lel: Laternula elliptica, contig00029.

for further investigation of gene and protein evolution in cold adapted metazoan species.

Animals living in constant cold temperatures could initially be thought to be more vulnerable to damage by reactive oxygen species, due to slow cell and protein turnover rates and the consequent accumulation of oxidised proteins $[12,65]$. However, L. elliptica is being used as a model for ageing studies as this organism appears to have uncoupled the ageing process and antioxidant production. It has a higher antioxidant capacity compared to shorter lived temperate clams, with a constant requirement for stable antioxidant status until old age $[11,12]$. Enzyme assays show that catalase, in particular, remains at a constant level throughout its lifetime and this is exemplified by the expression of catalase in the genome of adults of this species. The stress associated endoplasmic reticulum protein (SERP2) is also highly expressed and this may be linked to antioxidant capacity or it may protect unfolded target proteins against degradation and facilitate correct glycosylation. As a member of the RAMP4 family (ribosome associated membrane proteins) it may also be involved in the stabilisation of membranes in response to stress. It is known that there are problems folding proteins at low temperatures [66] and to date, of the few Antarctic marine species investigated, the majority do not exhibit the classical heat shock protein (HSP) stress response. Indeed, several species express the inducible form of HSP70 permanently, possibly as a measure towards more efficient folding of proteins at low temperatures [21] and the expression of SERP2 may contribute towards this "extra" required function and form part of a "preparative defence" strategy against the cold [67].

The final category of highly expressed genes comprises those involved in immune function, e.g. thymosin $\beta$ [68], $\alpha$ macroglobulin and a mannan-binding lectin associated serine protease, which is a complement control module. The reason for this up-regulation may be slightly more complex than it initially appears. The mantle edge is in constant contact with the external environment and hence there will be permanent challenges to the immune system. This is compounded in the Rothera population of L. elliptica by a significant amount of physical damage (Harper, pers comm). These almost certainly will require the enhanced expression of immune-related genes, as the external protection of the shell is compromised, along with matrix deposition for shell repair. However, physical damage is a stressor in its own right which along with the effect of the cold environment (another potential stressor) may induce changes in the immune system as stress and the immune system have been found in many species to be inextricably linked [69].

\section{Putative shell deposition transcripts}

The formation of the skeleton in animals is well conserved and frequently involves calcification of a macromolecular network of proteins, lipids and polysaccharides. In molluscs the mantle is the source of matrix proteins and other secreted factors which promote the extracellular assembly of the shell. Relatively few matrix proteins contributing to the shell in molluscs have been identified and most of the studies so far have focused on single proteins such as Asprich, lustrin A, perlustrin and calconectin, whilst other proteins involved in calcium deposition include carbonic anhydrase [36,3840,70-72]. In a recent study, 331 randomly selected clones from a cDNA library of the juvenile mantle of tropical abalone (Haliotis asinina, Linnaeus) were sequenced [73]. The authors reported that $26 \%$ of the genes encoded secreted proteins and of the 106 unigenes identified 15 were involved in trafficking and mineral binding, mechanisms which they suggested probably contribute to construction of the shell. In the present study a conservative 
estimate using the GO cellular component annotation of known genes suggests $40 \%$ of the transcripts are likely to be secreted proteins. A comparison of the transcriptome of the mantle from adult L. elliptica with the cDNA isolated from juvenile tropical abalone mantle [73] revealed relatively poor conservation, with only 31 of the Haliotis sequences sharing significant sequence similarity with the Laternula transcripts. This may be due to either the disparity in sample sizes or maturity stage of the animals, rather than evolutionary distance, as BLAST sequence similarity searching of all 6778 Haliotis asinina sequences in GenBank produced a higher match with 728 Laternula contigs matching 1435 Haliotis sequences (21\%). Indeed there were relatively few matches to ESTs from libraries generated specifically to study nacre building gene sets in Haliotis asinina and the bivalve Pinctada maxima (6,122 and 6,737 ESTs respectively) indicating the divergence in biomineralisation processes between these two different molluscs [56]. This was further highlighted in the Haliotis/Pinctada study, where there was very little overlap between even the most highly expressed genes and addition of the results from the Laternula and M. galloprovincialis datasets substantiate this (Table 2). Hence there is a requirement to understand shell deposition in a variety of molluscs and not just work on a single model species, particularly where there is a requirement to understand environmental effects.

Several of the most highly expressed genes in our dataset are almost certainly involved in shell deposition, including tyrosinase. The periostracum is secreted as a soluble precursor (the periostracin) and this is then cross-linked by o-diphenols and tyrosinase (or phenoloxidases) to form an insoluble periostracum $[74,75]$. Tyrosinase can also be involved in pigment formation in the prismatic layer and evidence from the pearl oyster demonstrates several different paralogues of tyrosinase which are involved in these different functions [76,77]. However, in order to discover genes within our dataset that are likely to play a role in shell deposition and calcium regulation, we searched the literature to generate an inhouse database of proteins involved in extracellular matrix (ECM) formation and calcium homeostasis in metazoans (Supplemental Tables 1 and 2). Numerous transcripts were identified; hence the following section will give only a brief outline of the putative role of the more abundant transcripts.

The presence of putative transcripts for carbonic anhydrase in L. elliptica mantle is unsurprising as this protein was first identified in the shell in 1948 [78] and it has subsequently been implicated in matrix mineralisation by generating an acidic environment through the conversion of respiratory $\mathrm{CO}_{2}$ into $\mathrm{HCO}_{3}$ in the presence of water [38]. Putative transcripts for the matricellular glycoprotein, secreted protein acidic rich in cystein (SPARC, a basal membrane component) were also identified. This trimodular protein promotes proper assembly and maturation of the matrix scaffold and is highly conserved in animal phyla [79]. In vertebrates the latter is achieved in part through the interaction of SPARC with fibril forming collagens (I, II, III and V) $[80,81]$ and although it is necessary to conduct further work to better characterize these transcripts, orthologues of collagen I, II and V were identified.

Additional transcripts identified in the L. elliptica manthe transcriptome potentially implicated in ECM formation/turnover in metazoans include the thrombospondins, which are a family of large, secreted, multi-modular, calcium-binding glycoproteins which appear to interact with collagens and integrins and have been implicated in skeletal disorders in mammals [82]. Transcripts for tenascin, a large glycoprotein containing several fibronectin III type repeats and implicated in cell adhesion in chordates was identified in L. Elliptica [83]. Interestingly, several transcripts for metalloproteinase 1 (collagenase 1) which are important in extracellular matrix turnover were also identified. It is apparent even from this brief consideration that numerous homologues of genes identified in the ECM of the vertebrate skeleton are also present in the mantle transcriptome. Future work will permit a more precise characterization of the localization and function of these mantle transcripts and hence provide a better understanding of shell formation. This will be essential for ecophysiological studies.

\section{The clam orphan calcium regulatory receptors}

The L. elliptica contig11573 (283 bp) and contig14182 (252 bp) nucleotide reads share the highest sequence similarity for the N-terminal region and TM2 to TM3 of the metazoan parathyroid hormone receptor (PTHR) and calcitonin/calcitonin Gene-related peptide (CLR/ CGRPR) receptors, repectively (Table 3). In vertebrates these receptors are important mediators of the action of the calcitropic factors, calcitonin $(\mathrm{CT})$ and parathyroid hormone (PTH) which stimulate respectively, calcium uptake and bone formation and calcium release for serum and bone turnover. In invertebrates, putative protostome CLR/CGRPR transcripts that remain to be functionally characterised have previously been identified $[47,84]$. Several scaffolds were identified in the Lottia genome assembly which shared high sequence similarity $\left(\mathrm{e}^{-18}-\mathrm{e}^{-}\right.$ 13) to the L. elliptica contigs similar to PTHR/CLR. In molluscs, a CLR/CGRP, expressed in the mantle of the eastern oyster Crassostrea virginica (JC8022) [85] has been isolated and found to be functionally conserved with the vertebrate orthologues. Despite the recent identification of a prototype of vertebrate CT/CGRP peptide ligand in the ascidian Ciona intestinalis [86] no transcripts which are orthologues of $\mathrm{CT}$ or PTH have been 
Table 2: The ten most commonly expressed sequences (in order of abundance) in mantle tissue from 4 bivalves.

\begin{tabular}{|c|c|c|c|}
\hline Laternula elliptica & Haliotis asinina & Pinctada maxima & Mytilus galloprovincialis \\
\hline $\begin{array}{l}\text { map kinase interacting serine threonine } \\
\text { protein kinase. }\end{array}$ & - & shematrin & - \\
\hline collagen pro- $a$ chain & Elongation factor-1a & shematrin & - \\
\hline enolase & cytochrome c oxidase 1 & 16 s ribosomal protein & Phospholipase \\
\hline- & - & KRMP-8, glycine rich structural protein & - \\
\hline- & ferritin & Elongation factor-1a & NADH dehydrogenase subunit 4 \\
\hline- & collagen & actin & - \\
\hline ATP synthase sub-unit a & cytochrome c oxidase 1 & KRMP-8, glycine rich structural protein & - \\
\hline collagen type IV a6 & - & N14 matrix protein & NADH dehydrogenase subunit 4 \\
\hline troponin T & - & - & NADH dehydrogenase subunit 4 \\
\hline- & - & paramyosin & NADH dehydrogenase subunit 4 \\
\hline
\end{tabular}

identified in Laternula and the functional significance of the Antarctic bivalve receptors remains to be established.

Despite the relatively short sequence of the family 2 B1 G-protein coupled receptor transcripts in L. elliptica it was possible to identify conserved amino acid motifs implicated in receptor conformation and ligand affinity in metazoan orthologues (Figures 4 and 5) [84]. The characteristic $\mathrm{N}$-terminal motif for interacting with receptor activity-modifying proteins (RAMPs) was identified in contig11573 [87]. RAMPs are known to modulate GPCRs and in vertebrates they define the specificity of the CLR by modifying its affinity for the ligand. Putative RAMP transcripts were not identified in the present study and it remains to be established if the bivalve family 2 B1
GPCRs are modulated in a similar way to those in vertebrates.

\section{Conclusions}

Comprehensive 454 pyrosequencing of mantle tissue from the Antarctic clam (Laternula elliptica) has produced a transcriptome of 18,290 contigs. In spite of a low level of putative gene identifications $(17 \%$, produced via database sequence similarity searching), it was possible to identify a considerable number of transcripts putatively related to shell deposition (e.g. tyrosinase and SPARC). This was via similarity to annotated sequences from other molluscs in the databases or to genes known to be involved in skeletal formation in vertebrates. In particular, 2 putative members of family 2 GPCRs were identi-

Table 3: Best database matches of the Laternula sequences to the family 2 GPCRs.

\begin{tabular}{lcl}
\hline Read & Length (bp) & Sequence similarity \\
\hline contig11573 & 283 & $\begin{array}{l}\text { PREDICTED: similar to parathyroid hormone receptor [Nasonia vitripennis] 4e-17 calcitonin receptor [Culex } \\
\text { quinquefasciatus] 2e-14 }\end{array}$ \\
\hline contig14182 & 252 & PREDICTED: similar to calcitonin receptor, partial [Acyrthosiphon pisum] 9e-10 \\
\hline
\end{tabular}




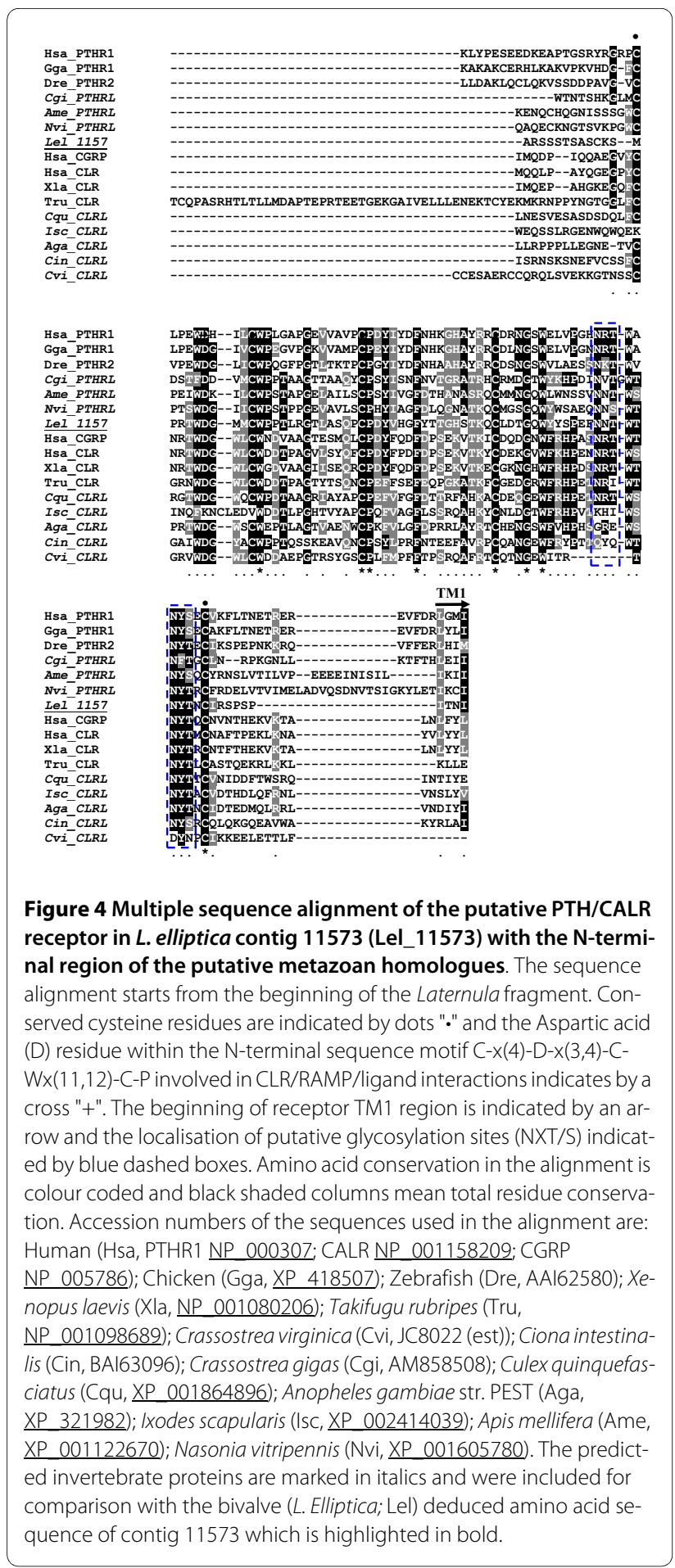

fied which share the highest sequence similarity to the metazoan parathyroid hormone (PTH) and calcitonin/ calcitonin Gene-related peptide (CLR/CGRPR) receptors. In vertebrates these genes are important mediators of the action of the calcitropic factors, calcitonin (CT) and parathyroid hormone (PTH). This dataset, whilst being the first 454 sequence to be generated from an Ant-

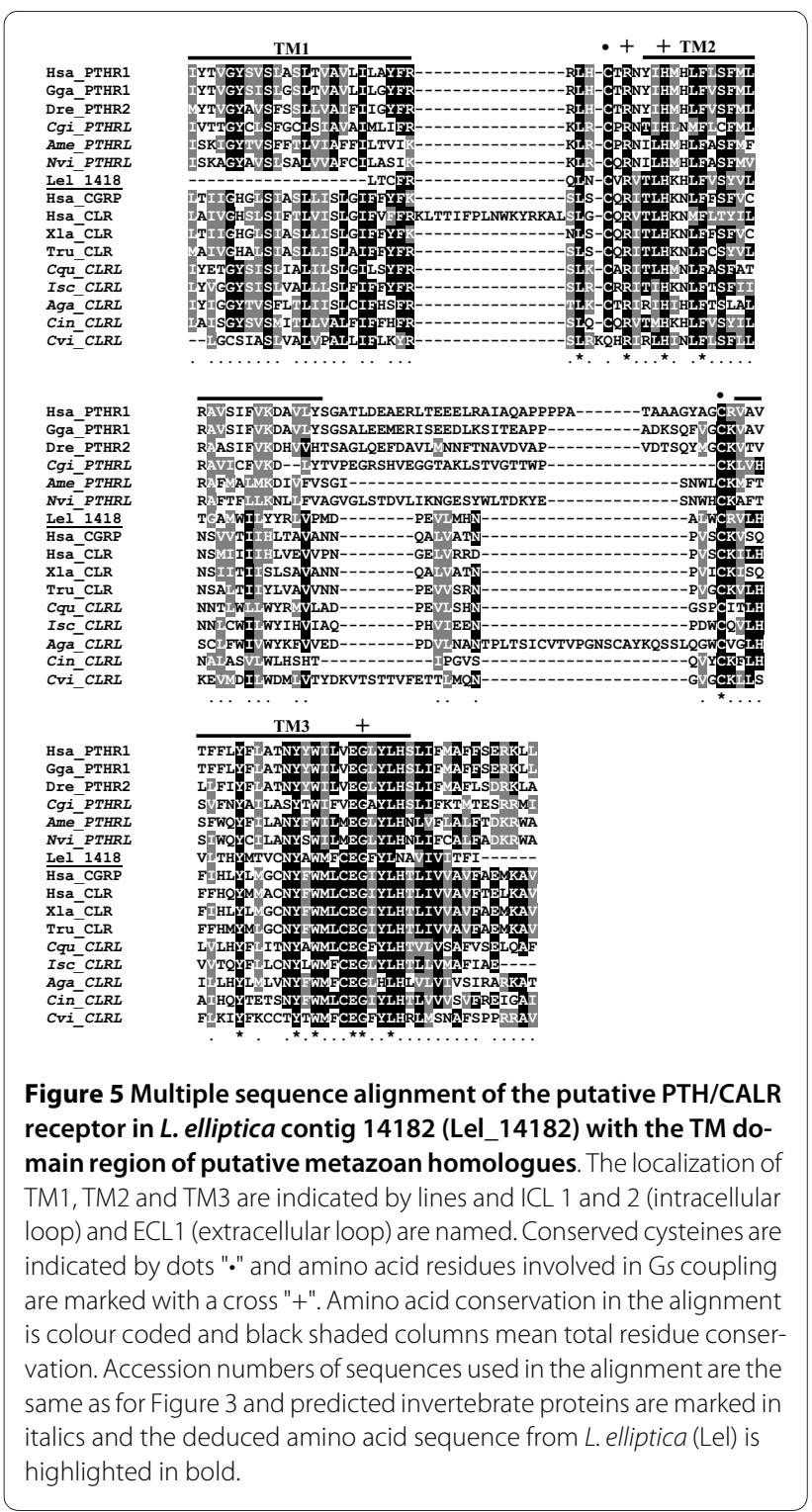

arctic invertebrate, also provides a significant resource for comparative studies into protein cold adaptation and the processes of shell deposition, in particular the reaction of the shell secretome to environmental stress in the form of climate change effects. Both avenues will be explored in the future within our laboratory, both in Laternula and economically important model temperate mollusc species, such as Crassostrea gigas and Mytilus galloprovincialis.

\section{Methods \\ Animal sampling}

All animals used in experimental work were collected at Rothera Research Station, Adelaide Island, Antarctic Peninsula ( $\left.67^{\circ} 4^{\prime} 07^{\prime \prime} \mathrm{S}, 68^{\circ} 07^{\prime} 30^{\prime \prime} \mathrm{W}\right)$ by SCUBA divers during the austral summer at depths of 10-15 m. The animals 
were immediately returned to the laboratory where they were maintained in a through-flow aquarium with a temperature of $0.6 \pm 0.3^{\circ} \mathrm{C}$, under a simulated natural light:dark cycle. All animals were mature adults, with a range of shell sizes between $50.1-83.5 \mathrm{~mm}$. As shell length is related to animal age: surface aging estimates using growth rings produced an mean age of 8.3 years (SE mean 0.207 ) with a range from 6-14 years and a median of 8 years (S. Morley pers comm). Mantle tissue was dissected from the animals and cross sections comprising all 3 folds and the periostracum were immediately flash frozen in liquid nitrogen for later RNA extraction.

\section{RNA isolation and CDNA production}

Mantle RNA was extracted from 24 animals using a modified TRI reagent protocol. After homogenization in Tri Reagent (Sigma) and chloroform extraction, the samples were subjected to a lithium chloride precipitation step. RNA was precipitated using a 1:1 isopropanol:saline solution $(0.8 \mathrm{M}$ sodium citrate and $1.2 \mathrm{M} \mathrm{NaCl})$ and after resuspension, the RNA was subjected to a further precipitation using $250 \mu \mathrm{l} 7.5 \mathrm{M} \mathrm{LiCl}$. The extractions were further cleaned using RNeasy mini kit columns (Qiagen, Crawley, Sussex, UK) following manufacturer instructions in order to eliminate $\mathrm{LiCl}$ and salt residues. $5 \mu \mathrm{g}$ of RNA was PCR amplified using the protocol described in [88] prior to preparation for the 454 run. Samples were nebulised at 30psi for one minute and subsequently purified with Ampure (Agencourt) to produce fragments 300 bp and above. The ends were polished and the 454 titananium adapters containing specific MID sequences were attached. Fragments containing both a and b adapters were selected and quantified. Libraries were amplified by emulsion PCR, beads recovered and enriched and placed on a picotiter plate for sequencing by the 454 procedure.

\section{Assembly and Analysis}

The raw data comprised 1,034,155 reads. Crossmatch (P. Green, unpublished) was then applied to screen for adaptor sequences and other artifacts of the pyrosequencing procedure and also vector sequences using the UniVec database http://www.ncbi.nlm.nih.gov/VecScreen/UniVec.html. Stripping the masked sequence from the ends and removing reads with masked sequence in the middle resulted in 778,629 sequences that were entered into the Newbler program [51] for assembly. This resulted in 18,290 contigs. All singletons were discarded. Files containing the reads have been submitted to the National Center for Biotechnology Information Short Read Archive (accession number SRA011054). The mapping facility of Newbler was applied to the assembly to determine the number of SNPs, and Phobos [89] was used for microsatellite discovery. The contigs were then searched for sequence similarity using BLAST [90] against the gen- bank non-redundant database [53] and unannotated data from other bivalve species: the gastropod snail: Lottia gigantea http://genome.jgi-psf.org/Lotgi1/ Lotgi1.home.html and the Mytilus 454 mantle-specific datasets (4442949.3: M. galloprovincialis mantle unassembled and 4442954.3 M. edulis mantle unassembled) [54] lodged under the MG-RAST database: Meta Genome Rapid Annotation using Sub-system Technology http://metagenomics.nmpdr.org/) [91]. The Gene Ontology (GO) [92] mappings were determined by an in-house database on all Swissprot and Trembl [93] BLAST scores below a threshold of 1e-10. Sequence manipulation was carried out using the EMBOSS suite of programmes [94]. Sequences were clustered using ClustalW [95] and the alignments displayed using BoxShade v3.21 [96].

\section{Additional material}

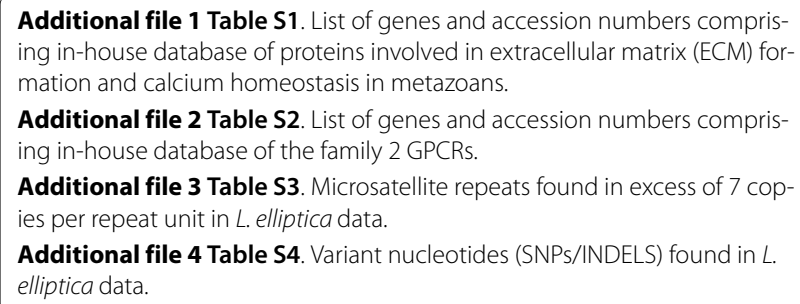

Additional file 1 Table S1. List of genes and accession numbers comprising in-house database of proteins involved in extracellular matrix (ECM) formation and calcium homeostasis in metazoans.

Additional file $\mathbf{2}$ Table S2. List of genes and accession numbers comprising in-house database of the family 2 GPCRs

Additional file 3 Table S3. Microsatellite repeats found in excess of 7 copies per repeat unit in L. elliptica data.

Additional file 4 Table S4. Variant nucleotides (SNPS/INDELS) found in L. elliptica data.

\section{Authors' contributions}

MSC progressed the original project idea through to 454 analysis, organised the sequencing, provided the manual assembly verification, analysis of the most commonly expressed transcripts and wrote this section of the manuscript, along with developing the initial concept of the paper and co-ordinating input for the final manuscript. MAST performed all the 454 computational analysis, including installation, development of the 454 pipeline and collation/ liaison of datasets. JCRC, FAV and DMP developed and produced the in-house databases on genes putatively involved in calcium regulation and shell deposition and the family 2 GPCRs. JCRC specifically analysed the family 2 GPCR data and FAV analysed the calcium regulation and shell deposition transcripts. DMP supervised this part of the analysis and wrote the section of the manuscript specifically relating to this, providing additional input into final editing of the manuscript. LSP assisted with development of the initial project concept, provided laternula material, physiological input and was involved in data interpretation, manuscript preparation and editing. All authors have read and approved the final manuscript.

\section{Acknowledgements}

This paper was produced within the BAS Q4 BIOREACH/BIOFLAME core programmes. The authors would like to thank Guy Hillyard for performing the RNA extractions, Gavin Burns for the PCR amplifications, Simon Morley for obtaining sample material and all members of the Rothera Dive Team for collecting animals. Overall diving support was provided by the NERC National Facility for Scientific Diving at Oban. The sequencing was performed at the NERC

Biomolecular Analysis Facility, Liverpool and we would particularly like to thank Margaret Hughes and Kevin Ashelford for their technical support. We would also like to thank Bela Tiwari of the NERC environmental Bioinformatics Centre (NEBC), CEH, Wallingford for valuable discussions on 454 software, analysis pipelines and processes and Elizabeth Harper at the University of Cambridge for advice on Laternula shell and mantle structure. Our analyses including searching against the Lottia gigantea sequence data v1.0 (July 24 2007) which was produced by the US Department of Energy Joint Genome Institute http:// www.jgi.doe.gov/ in collaboration with the user community and made publicly available via their web site: http://genome.jgi-psf.org/Lotgi1/ Lotgi1.home.html. 


\section{Author Details}

'British Antarctic Survey, Natural Environment Research Council, High Cross, Madingley Road, Cambridge, CB3 OET, UK and ${ }^{2}$ Center of Marine Sciences, Universidade do Algarve, Campus de Gambelas,8005-139 Faro, Portugal

Received: 22 February 2010 Accepted: 8 June 2010

Published: 8 June 2010

\section{References}

1. Morton B: The biology and functional morphology of Laternula truncata (Lamark 1818) (Bivalvia: Anomalodesmata: Pandoracea). Biol Bull 1973, 145:509-531.

2. Ahn I-Y: Ecology of the Antarctic bivalve Laternula elliptica (King and Broderip) in Collins Harbour, King George Island: benthic environment and adaptive strategy. Mem Natl Inst Polar Res Spec Issue 1994, 50:1-10.

3. Powell DK: The reproductive ecology of Antarctic free-spawning molluscs. PhD thesis, University of Southampton, Southampton, UK; 2001:142.

4. Kang DH, Ahn IY, Choi KS: The annual reproductive pattern of the Antarctic clam, Laternula elliptica from Marian Cove, King George Island. Polar Biol 2009, 32:517-528.

5. Ansell AD, Harvey R: Protected larval development in the Antarctic bivalve Laternula elliptica (King and Broderip) (Anomalodesmata: Laternulidae). J Molluscan Stud 1997, 63:285-286.

6. Peck LS, Powell DK, Tyler PA: Very slow development in two Antarctic bivalve molluscs, the infaunal clam Laternula elliptica and the scallop Admussium colbecki. Mar Biol 2006, 150:1191-1197.

7. Ralph R, Maxwell JGH: Growth of 2 Antarctic Lamellibranchs Adamussium colbecki and Laternula elliptica. Mar Biol 1977, 42:171-175.

8. Brey T, Mackensen A: Stable isotopes prove shell growth bands in the Antarctic bivalve Laternula elliptica to be formed annually. Polar Biol 1997, 17:465-468.

9. Brockington S: The seasonal energetics of the Antarctic bivalve Laternula elliptica (King and Broderip) at Rothera Point, Adelaide Island. Polar Biol 2001, 24:523-530.

10. Morley SA, Peck LS, Miller AJ, Pörtner HO: Hypoxia tolerance associated with activity reduction is a key adaptation for Laternula elliptica seasonal energetics. Oecologia 2007, 153:29-36.

11. Abele D, Brey T, Philipp E: Bivalve models of aging and the determination of molluscan lifespans. Exp Gerontol 2009, 44:307-315.

12. Philipp E, Pörtner HO, Abele D: Mitochondrial ageing of a polar and a temperate mud clam. Mech Ageing Dev 2005, 126:610-619.

13. Peck LS, Pörtner HO, Hardewig I: Metabolic demand, oxygen supply, and critical temperatures in the Antarctic bivalve Laternula elliptica. Physiol Biochem Zool 2002, 75:123-133.

14. Peck LS, Webb KE, Bailey DM: Extreme sensitivity of biological function to temperature in Antarctic marine species. Func Ecol 2004, 18:625-630.

15. Peck LS, Morley SA, Pörtner HO, Clark MS: Thermal limits of burrowing capacity are linked to oxygen availability and size in the Antarctic clam Larenula elliptica. Oecologia 2007, 154:479-484.

16. Peck LS, Clark MS, Morley SA, Massey A, Rosetti H: Animal temperature limits: effects of size, activity and rates of change. Func Ecol 2009, 23:248-256

17. Murphy JM, Mitchell JFB: Transient-response of the Hadley Center coupled ocean-atmosphere model to increasing carbon-dioxide. 2. Spatial and temporal structure of response. J Climate 1995, 1:57-80.

18. IPCC: Climate change 2007: synthesis report. In Contribution of work groups I, II and III to the 4th Assessment Report of the Intergovernmental Panel on Climate Change. Core writing team Edited by: Pachauri RK, Reisinger A. IPCC, Geneva, Switzerland; 2007.

19. Meredith MP, King JC: Rapid climate change in the ocean west of the Antarctic Peninsula during the second half of the 20th century. Geophys Letts 2005, 32:L19604-L19609.

20. Barnes DKA, Peck LS: Is Antarctic shelf biodiversity vulnerable to predicted regional warming? Climate Res 2008, 37:149-163.

21. Clark MS, Peck LS: HSP70 Heat shock proteins and environmental stress in Antarctic marine organisms: a mini-review. Mar Gen 2009, 2:11-18.

22. Ahn I-Y, Lee SH, Kim KT, Shim JH, Kim DY: Baseline heavy metal concentrations in the Antarctic clam Laternula elliptica in Maxwell Bay, King George Island, Antarctica. Mar Pollut Bull 1996, 32:592-598.

23. Dell RK: Antarctic benthos. Adv Mar Biol 1972, 10:1-216.
24. Picken GB: Marine habitats: Benthos. In Key Environments - Antarctica Edited by: Bonner WN, Walton DWH. Pregamon Press, Oxford; 1985:P154-172.

25. Arntz WE, Brey T, Gallardo VA: Antarctic zoobenthos. Oceanogr Mar Biol Ann Rev 1994, 32:241-304.

26. Ahn I-Y: Feeding ecology of the Antarctic bivalve Laternula elliptica (Latinulidae) in Marian Cove and vicinity, King George Island during one austral summer. In Antarctic Communities: Species, structure and survival Edited by: Battaglia B, Valencia J, Walton DW. Cambridge University Press, Cambridge; 1997:142-151.

27. Caldeira K, Wickett ME: Anthropogenic carbon and ocean $\mathrm{pH}$. Nature 2003, 425:365-365.

28. Caldeira K, Wickett ME: Ocean model predictions of chemistry changes from carbon dioxide emissions to the atmosphere and ocean. Geophys Res 2005, 110:C9.

29. Royal Society. 2005. Policy document 12/05

30. Cao L, Caldeira K, Jain AK: Effects of carbon dioxide and climate change on ocean acidification and carbonate mineral saturation. Geophys Res Letts 2007, 34:5

31. Orr JC, Fabry VJ, Aumont O, Bopp L, Doney SC, Feely RA, Gnanadesikan A, Gruber N, Ishida A, Joos F, Key RM, Lindsay K, Maier-Reimer E, Matear R, Monfray P, Mouchet A, Naijar RG, Plattner G-K, Rodgers KB, Sabine CL, Sarmiento IL, Schlitzer R, Slater RD, Totterdell IJ, Weirig M-F, Yamanaka Y, Yool A: Anthropogenic ocean acidification over the twenty-first century and its impact on calcifying organisms. Nature 2005, 437:681-686.

32. McLachlan A, Jaramillo E, defeo O, Dugan J, de Ruyck A, Coetzee P. Adaptations of bivalves to different beach types. J Exp Mar Biol Ecol 1995, 187:147-160.

33. Hoffman Jl, Peck LS, Hillyard G, Zieritz A, Clark MS: No evidence for genetic differentiation between Antarctic limpet Nacella concinna morphotypes. Mar Biol 2010, 157:765-778.

34. McClintock JB, Angus RA, McDonald MR, Amsler CD, Catledge SA, Vohra YK: Rapid dissolution of shells of weakly calcified Antarctic benthic macroorganisms indicates high vulnerability to ocean acidification. Antarct Sci 2009, 21:449-456.

35. Addadi L, Weiner S: Interactions between acidic proteins and crystals: Stereochemical requirements in biomineralization. Proc Natl Acad Sci USA 1985, 82:4110-4114.

36. Duplat D, Puissegur M, Bedouet L, Rousseau M, Boulzaguet H, Milet C, Sellos D, Van Wormhoudt A, Lopez E: Identification of calconectin, a calcium-binding protein specifically expressed by the mantle of Pinctada margaritifera. FEBS Lett 2006, 580:2435-2441.

37. Marin F, Luquet G: Molluscan shell proteins. Comptes Rendus Palevol 2004, 3:469-492

38. Miyamoto H, Miyashita T, Okushima M, Nakano S, Morita T, Matsushiro A A carbonic anhydrase from the nacreous layer in oyster pearls. Proc Natl Acad SciUSA 1996, 93:9657-9660.

39. Shen XY, Belcher AM, Hansma PK, Stucky GD, Morse DE: Molecular cloning and characterization of lustrin $\mathrm{A}$, a matrix protein from shell and pearl nacre of Haliotis rufescens. J Biol Chem 1997, 272:32472-32481.

40. Weiss IM, Gohring W, Fritz M, Mann K: Perlustrin, a Haliotis laevigata (Abalone) nacre protein, is homologous to the insulinlike growth factor binding protein $\mathrm{N}$-terminal module of vertebrates. Biochem Biophys Res Comm 2001, 285:244-249.

41. Barrera E, Tevesz MJS, Carter JC, McCall PL: Oxygen and carbon isotopic composition and shell microstructure of the bivalve Larenula elliptica from Antarctica. Palaios 1994, 9:275-287.

42. Harper EM: The molluscan periostracum: an important constraint in bivalve evolution. Palaeontology 1997, 40:71-97.

43. Park H, Ahn IY, Kim H, Cheon J, Kim M: Analysis of ESTs and expression of two peroxiredoxins in the thermally stressed Antarctic bivalve Laternula elliptica. Fish Shellfish Immunol 2008, 25:550-559.

44. Kim M, Ahn IY, Kim H, Cheon J, Park H: Molecular characterization and induction of heat shock protein 90 in the Antarctic bivalve Laternula elliptica. Cell Stress Chaperones 2009, 14:363-370.

45. Clark MS, Fraser KPP, Peck LS: Antarctic marine molluscs do have an HSP70 heat shock response. Cell Stress Chaperones 2008, 13:39-49.

46. Power DM, Ingleton PM, Clark MS: The application of comparative genomics to fish endocrinology. Internat Rev Cyto/ 2002, 221:149-190.

47. Cardoso JCR, Pinto WV, Vieira FA, Clark MS, Power DM: Evolution of secretin family GPCR members in metazoa. BMC Evol Biol 2006, 6:108. 
48. Cardoso JCR, de Vet ECJM, Louro B, Elgar G, Clark MS, Power DM: Persistence of duplicated PAC1 receptors in the teleost, Sparus aurata. BMC Evol Biol 2006, 7:221.

49. Ellegren $\mathrm{H}$ : Sequencing goes 454 and takes large-scale genomics into the wild. Mol Ecol 2008, 17:1629-1631

50. Meyer E, Aglyamova GV, Wang S, Buchanan-Carter J, Abrego D, Colbourne JK, Willis BL, Matz MV: Sequencing and do novo analysis of a coral larval transcriptome using 454 GSFIx. BMC Genomics 2009, 10:219.

51. [http://www.454.com]

52. Hahn DA, Ragland GJ, Shoemaker DD, Denlinger DL: Gene discovery using massively parallel pyrosequencing to develop ESTs for the flesh fly Sarcophaga crassipalpis. BMC Genomics 10:234.

53. Benson DA, Karsch-Mizrachi I, Lipman DJ, Ostell J, Wheeler DL: GenBank. Nucl Acids Res 2007, 35:D21-25

54. Craft JA, Gilbert JA, Temperton B, Dempsey KE, Ashelford K, Tiwari B, Hutchinson TH, Chipman JK: Pyrosequencing of Mytilus galloprovincialis cDNAs: Tissue-specific expression patterns. PlosOne 2010, 5:e8875.

55. Hooper SL, Thuma JB: Invertebrate muscles: Muscle specific genes and proteins. Physiol Rev 2005, 85:1001-1060.

56. Jackson DJ, McDougall C, Woodcroft B, Maose P, Rose RA, Kube M, Reinhardt R, Rokhsar DS, Montagnani C, Joubert C, Piquemal D, Degnan BM: Parallel evolution of nacre building gene sets in molluscs. Mol Biol Evol 2010, 27:591-608

57. Pyronnet $\mathrm{S}$, Imataka H, Gingras AC, Fukunaga $\mathrm{R}$, Hunter T, Sonenberg N: Human eukaryotic translation initiation factor 4G (elF4G) recruits Mnk1 to phosphorylate elF4E. Embo J 1999, 18:270-279.

58. Ross G, Dyer JR, Castellucci VF, Sossin WS: Mnk is a negative regulator of cap-dependent translation in Aplysia neurons. J Neurochem 2006, 97:79-91.

59. Waskiewicz AJ, Flynn A, Proud CG, Cooper JA: Mitogen-activated protein kinases activate the serine/threonine kinases Mnk1 and Mnk2. Embo J 1997, 16:1909-1920.

60. Kloks CPAM, Spronk CAEM, Lasonder E, Hoffmann A, Vuister GW, Grzesiek S, Hilbers CW: The solution structure and DNA-binding properties of the cold-shock domain of the human Y-box protein YB-1. J Mol Biol 2002, 316:317-326.

61. Canonaco F, Schlattner U, Pruett PS, Wallimann T, Sauer U: Functional expression of phosphagen kinase systems confers resistance to transient stresses in Saccharomyces cerevisiae by buffering the ATP pool. J Biol Chem 2002, 277:31303-31309.

62. Kucharski R, Maleszka R: Arginine kinase is highly expressed in the compound eye of the honey bee, Apis mellifera. Gene 1998, 211:343-349.

63. Tanaka K, Uda K, Shimada M, Takahashi Kl, Gamou S, Ellington WR, Suzuki T: Evolution of the cytoplasmic and mitochondrial phosphagen kinases unique to annelid groups. J Mol Evol 2007, 65:616-625

64. Römisch K, Collie N, Soto N, Logue J, Lindsay M, Scheper W, Cheng C-HC: Protein translocation across the endoplasmic reticulum membrane in cold-adapted organisms. J Cell Sci 2003, 116:2875-2883.

65. Fraser KPP, Clarke A, Peck LS: Growth in the slow lane: protein metabolism in the Antarctic limpet Nacella concinna (Strebel, 1908). J Exp Biol 2007, 210:2691-2699.

66. Privalov PL: Cold denaturation of proteins. Crit Rev Biochem Mol Biol 1990, 25:281-305.

67. Dong Y, Miller LP, Sanders JG, Somero GN: Heat-Shock Protein 70 (Hsp70) expression in four limpets of the Genus Lottia: Interspecific variation in constitutive and inducible synthesis correlates with in situ exposure to heat stress. Biol Bull 2008, 215:173-181.

68. Wu L, Wu X: Molecular cloning and expression analysis of $a$ b-thymosin homologue from a gastropod abalone, Haliotis diversicolor supertexta. Fish Shellfish Immunol 2009, 27:379-382.

69. Padgett DA, Glaser R: How stress influences the immune response. Trends Immunol 2003, 24:444-448.

70. Gotliv BA, Kessler N, Sumerel JL, Morse DE, Tuross N, Addadi L, Weiner S: Asprich: A novel aspartic acid-rich protein family from the prismatic shell matrix of the bivalve Atrina rigida. Chembiochem 2005, 6:304-314.

71. Mann K, Weiss IM, Andre S, Gabius H-J, Fritz M: The amino-acid sequence of the abalone (Haliotis laevigata) nacre protein perlucin. Detection of a functional C-type lectin domain with galactose/mannose specificity. Eur J Biochem 2000, 267:5257-5264.
72. Samata T, Hayashib N, Konoa M, Hasegawac K, Horitad C, Akerad S: A new matrix protein family related to the nacreous layer formation of Pinctada fucata. FEBS Lett 1999, 462:225-229.

73. Jackson DJ, McDougall C, Green K, Simpson F, Wörheide G, Degnan BM: A rapidly evolving secretome builds and patterns a sea shell. BMC Biology 2006, 4:40.

74. Waite JH, Saleuddin ASM, Andersen SO: Periostracin- a soluble precursor of sclerotized periostracum in Mytilus edulis L. J Comp Physiol B 1979 130:301-307.

75. Saleuddin ASM, Petit HP: The mode of formation and the structure of the periostracum. In The Mollusca, Physiology Volume 4. Edited by: Saleuddin ASM, Wilbur KM. Academic Press, NewYork; 1983:199-233.

76. Zhang C, Xie L, Huang J, Chen L, Zhang R: A novel putative tyrosinase involved in periostracum formation from the pearl oyster (Pinctada fucata). Biochem Biophys Res Comm 2006, 342:632-639.

77. Nagai K, Yano M, Morimoto K, Miyamoto H: Tyrosinase localization in mollusc shells. Comp Biochem Physiol B 2007, 146:207-214.

78. Freeman JA, Wilbur KM: Carbonic anhydrase in molluscs. Biol Bull 1948, 94:55-59.

79. Koehler A, Desser S, Chang B, MacDonald J, Tepass U, Ringuette M: Molecular evolution of SPARC: absence of the acidic module and expression in the endoderm of the starlet sea anemone, Nematostella vectensis. Dev Genes Evol 2009, 219:509-521.

80. Sasaki T, Hohenester E, Gohring W, Timpl R: Crystal structure and mapping by site directed mutagenesis of the collagen binding epitope of an activated form of BM-40/SPARC/osteonectin. EMBO J 1998, 17:1625-1634

81. Sweetwyne MT, Brekken RA, Workman G, Bradshaw AD, Carbon J, Siadak AW, Murri C, Sage EH: Functionnal analysis of the matricellular protein SPARC with novel monoclonal antibodies. J Histochem Cytochem 2004 52:723-733

82. Posey LK, Hankenson K, Veerisetty AC, Bornstein P, Lawler J, Hecht Jacqueline T: Skeletal abnormalities in mice lacking extracellular matrix proteins, thrombospondin-1, thrombospondin-3, thrombospondin-5, and type IX collagen. Am J Pathol 2008, 172:664-1674.

83. Tucker RP, Drabikowski K, Hess JF, Ferralli J, Chiquet-Ehrismann R, Adams $J C$ : Phylogenetic analysis of the tenascin gene family: evidence of origin early in the chordate lineage. BMC Evol Biol 2006, 6:60.

84. Harmar AJ, Arimura A, Gozes I, Journot L, Laburthe M, Pisegna JR, Rawlings SR, Robberecht P, Said SI, Sreedharan SP, Wank SA, Waschek JA: International Union of Pharmacology. XVIII. Nomenclature of receptors for vasoactive intestinal peptide and pituitary adenylate cyclaseactivating polypeptide. Pharmacol Rev 1998, 50:265-270.

85. Dubos M-P, Badariotti F, Rodet F, LeLong C, Favrel P: Molecular and physiological characterization of an invertebrate homologue of a calcitonin-related receptor. Biochem Biophys Res Comm 2003, 310:972-978.

86. Sekiguchi T, Suzuki N, Fujiwara N, Aoyama M, Kawada T, Sugase K, Murata Y, Sasayama Y, Ogasawara M, Satake H: Calcitonin in a protochordate, Ciona intestinalis - the prototype of the vertebrate calcitonin/calcitonin gene-related peptide superfamily. FEBS J 2009, 276:4437-4447.

87. Ittner LM, Luessi F, Koller D, Born W, Fischer JA, Muff R: Aspartate ${ }^{69}$ of the calcitonin-like receptor is required for its functional expression together with receptor-activity-modifying proteins 1 and -2 . Biochem Biophys Res Comm 2004, 319:1203-1209.

88. Petalidis L, Bhattacharyya S, Morris GA, Collins VP, Freeman TC, Lyons PA Global amplification of mRNA by template-switching PCR: linearity and application to microarray analysis. Nucleic Acids Res 2003, 31:e142.

89. Mayer C: Phobos 3.3.10 2006 [http://www.rub.de/spezzoo/cm/ $\mathrm{cm}$ phobos.htm]

90. Altschul SF, Madden TL, Schaffer AA, Zhang JH, Zhang Z, Miller W, Lipman DJ: Gapped BLAST and PSI-BLAST: a new generation of protein database search programs. Nucl Acids Res 1997, 25:3389-3402.

91. Meyer F, Paarmann D, D'Souza M, Olson R, Glass EM, Kubal M, Paczian T, Rodriguez A, Stevens R, Wilke A, Wilkening J, Edwards RA: The Metagenomics RAST server - A public resource for the automatic phylogenetic and functional analysis of metagenomes. BMC Bioinformatics 2008, 9:386.

92. The Gene Ontology Consortium: Gene Ontology: tool for the unification of biology. Nat Genet 2000, 25:25-29.

93. The UniProt Consortium: The Universal Protein Resource (UniProt). NuCl Acids Res 2007, 35:D193-197. 
94. Rice P, Longden I, Bleasby A: EMBOSS, the European Molecular Biology Open Software Suite. Trends Genet 2000, 16:276-277.

95. Higgins JD, Gibson TJ: ClustalW - improving the sensitivity of progressive multiple sequence alignment through weighting, position-specific gap penalties and weight matrix choice. Nucl Acids Res 1994, 22:4673-4680.

96. Boxshade [http://www.ch.embnet.org/software/BOX form.html]

doi: $10.1186 / 1471-2164-11-362$

Cite this article as: Clark et al., Insights into shell deposition in the Antarctic bivalve Laternula elliptica: gene discovery in the mantle transcriptome using 454 pyrosequencing BMC Genomics 2010, 11:362

Submit your next manuscript to BioMed Central and take full advantage of:

- Convenient online submission

- Thorough peer review

- No space constraints or color figure charges

- Immediate publication on acceptance

- Inclusion in PubMed, CAS, Scopus and Google Scholar

- Research which is freely available for redistribution

Submit your manuscript at www.biomedcentral.com/submit 